\title{
Optimization of Multiple Response Variables Using the Desirability Function and a Bayesian Predictive Distribution
}

\author{
Jorge Limon-Romero ${ }^{1}$, Yolanda Baez-Lopez ${ }^{1}$, Diego Tlapa ${ }^{1}$, Mitzy Pory-Lugo ${ }^{1}$, \\ Ivan Rodriguez-Borbon ${ }^{2}$ \\ ${ }^{1}$ Universidad Autónoma de Baja California, \\ Facultad de Ingeniería, Arquitectura y Diseño, Baja California, \\ Mexico \\ ${ }^{2}$ Universidad Autónoma de Ciudad Juarez, Chihuahua, \\ Mexico \\ \{jorge.limon, yolanda, diegotlapa, mitzy.pory.lugo\}@uabc.edu.mx, \\ Ivan.rodriguez@uacj.mx
}

\begin{abstract}
This paper proposes the modification of a technique of simultaneous optimization of multiple response variables that works using a Bayesian predictive distribution, to incorporate different weights to the response variables according to their importance in the products. To achieve this, the desirability function has been incorporated to the original proposal. This research shows by representing different scenarios in one case study taken from literature, that the highest desirabilities and in turn the proposed optimum values in the process operating conditions always moved toward regions where the response variables with the highest weights had the best results, at the expense of performance in variables with the lowest weights.
\end{abstract}

\section{Introduction}

When working with product optimization, they are likely to have several quality characteristics that must be considered in the analysis. An alternative is to optimize separately each one of these characteristics, which according to various authors is not recommended because this approach may propose as many different combinations of control variables as response variables are considered in the study, which could generate a conflict to decide between them. The best way to address this situation is to consider all responses simultaneously, for which various techniques have been proposed, some of them more complete than others in relation to the various considerations that must be addressed during optimization. It is possible to find alternatives with different levels of technical complexity, with some of them even included within commercial statistical software, which helps them to be the best known and therefore used, although not necessarily the best options. This paper presents a Bayesian approach proposed by [1] which considers the correlation between the responses of interest and the uncertainty in the estimation of the model parameters in a 
formal manner. Furthermore it is considered what was proposed by [2], to incorporate noise variables to the analysis. The contribution that will be made have a direct impact on optimizing multiple responses, since this is a modification that allows incorporate a different weights for each response variable, according to the importance they have on the functionality or cost of the product. This will be accomplished by replacing the probability of conformance as variable to optimize in the original approach by the overall desirability (OD) for every process operating condition being analyzed.

\subsection{Background}

As already mentioned, several options have emerged to work with the problem of simultaneous optimization of multiple responses, the most common based on the loss function [3][4] on process capability indexes [5][6], on the desirability function [7] [8] on the overlapping of graphical response surfaces and have recently emerged techniques that optimize the probability of concordance [9][1]. Some of these techniques emerged to overcome a weakness that had other options or even to complement its earlier versions evolving on the same idea and incorporating some changes that would make them more complete.

Some aspects to consider by the various alternatives proposed to carry out the simultaneous optimization of the response variables are the correlation between the response variables, they must also consider noise factors that affect the quality characteristics and propose operating conditions of the processes that are robust to them and also must incorporate uncertainty in model parameters, all of this without losing the simplicity. So the right alternative must then be selected considering some of these elements.

There have been many efforts by various researchers to design a tool that includes as many of the above mentioned aspects as possible and this information may be found on different research journals. This study proposes to modify an existing technique using the method proposed by Peterson [1] based on the Bayesian approach, incorporating the robust approach proposed by [2] and incorporating the possibility of adding weights to the response variables that presents the methodology based on the desirability function, thus obtaining a hybrid methodology that considers the Bayesian methodology and desirability function to take the benefits of both proposals, for the simultaneous optimization of multiple responses.

\subsection{Desirability Function}

The desirability function according to [10] is a technique used for optimization of multiple responses in the analysis of experiments in which multiple responses must be optimized simultaneously. According to [11], the desirability optimization methodology is based on the idea that the quality of a product or process having multiple features, when one of these is outside certain "desired" limits, it is completely unacceptable. The method tries to find process operating conditions that provide the "most desirable" response. The optimal values of the factors are determined from the maximization of the function. A high value of $\mathrm{D}$, which varies between zero and one, indicates the best combination of factors to optimize the system studied. [6] propose using desirability functions which converts the problem of multiple responses into a 
problem of a single response; that is, the response analyzed is the overall desirability $D=\left(d_{1}\left(Y_{1}\right) . . d_{m}\left(Y_{m}\right)\right)^{1 / m}$ where $\left(Y_{1}, \ldots, Y_{m}\right)$ are the $m$ responses and $d_{1}, \ldots, d_{m}$ are the individual desirabilities. To convert the response $d_{i}$ to $y_{i}$ these authors propose the following transformation:

$$
d_{i}= \begin{cases}{\left[\frac{\hat{y}_{i}(x)-E I_{i}}{T_{i}-E I_{i}}\right]^{s}} & E I_{i} \leq \hat{y}_{i}(x) \leq T_{i}, \\ {\left[\frac{\hat{y}_{i}(x)-E S_{i}}{T_{i}-E S_{i}}\right]^{t}} & T_{i} \leq \hat{y}_{i}(x) \leq E S_{i}, \\ 0 \quad \hat{y}_{i}(x)<E I_{i} & o \quad \hat{y}_{i}(x)>E S_{i},\end{cases}
$$

where $s$ and $t$ are used to choose the desired shape of the transformation and thus reflect the wishes of the experimenter: if large values are taken $(\mathrm{s}, \mathrm{t} \geq 10)$ means that the desirability $d_{i}$ only takes large values when it falls near its target value; small values for $\mathrm{s}$ and $\mathrm{t}(\mathrm{s}, \mathrm{t} \leq 0.1)$ means that any value $\hat{y}_{i}$ within the range $\left[\mathrm{LS}_{\mathrm{i}}, \mathrm{US}_{\mathrm{i}}\right]$ is also desirable. This can be seen in Figure 1.

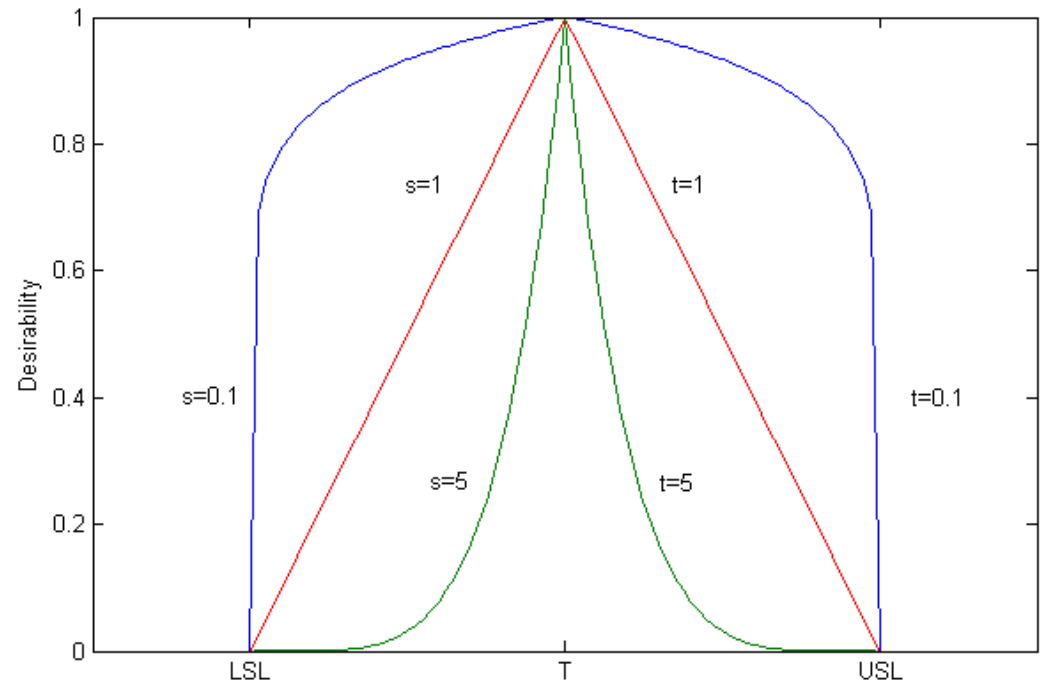

Fig. 1. Desirability function according to different values of $s$ and $t$.

According to [12] the default value of these exponents is one, suggesting linear increase desirability to the target value (see Figure 1). Also if a response variable has specification on only one side, what must be done is to take the target value (T) equal to the value at which it is considered that no additional gain on quality of the response 
is achieved, and in the equation 1 one restriction disappears and figure 1 , reduces to one side of the target value (T). According to [15] once the " $n$ " variables (levels of factors and responses) are transformed into values of desirability, these are combined into a single function called Overall Desirability (OD) to find the best set of responses using the following equation:

$$
\mathrm{OD}=\left(d_{1}^{r_{1}} \times d_{2}^{r_{2}} \times \ldots \times d_{n}^{r_{n}}\right)^{\frac{1}{\sum n}}=\left[\prod_{i=1}^{\mathrm{n}} d_{i}^{r_{i}}\right]^{\frac{1}{\sum n}}
$$

where $r_{i}$ refers to weights that represent the importance of each variable with respect to others.

To reach an OD different to zero, it is necessary that all the variables that are being optimized simultaneously have a desirability value greater than zero. On the other hand, if in any operating condition of the process, one of the responses is completely undesirable with $d_{i}\left(\hat{y}_{i}\right)=0, O D$ is also equal to zero, no matter how good the performance is in the remaining variables.

The optimization of multiple responses through desirability function according to [14] has two disadvantages: first, $O D$ can be difficult to model because it is a complex function of $m$ responses; second, it is difficult to say what the difference between expected values of $D$ means except that the bigger is the better. Another disadvantage of this approach according to [15] is that it does not consider the structure of variancecovariance matrix of responses and as it was previously mentioned ignore this information can lead to a no real solution, if indeed the responses have significantly different variances or if they are highly correlated. Other approaches have been proposed based on the desirability function to correct some of these details from the original proposal, see for example, [16, 7, 8, 17, 18].

\subsection{The Bayesian Approach}

According to [2] the optimization of multiple responses problem is to choose the values of $k$ controllable factors $x_{i}$, such that $\boldsymbol{Y}$ has certain desired properties. Often the case is presented in which these properties are the vector of responses $\boldsymbol{Y}$ meet specifications. Then let $\boldsymbol{A}$ represents the region of space defined by these specifications responses. Region $\boldsymbol{A}$ may have an arbitrary shape. Then optimizing multiple responses can be summarized in a simple goal, which is to maximize the probability of having the vector of responses within the specification region $\boldsymbol{A}$, for example, maximize the following probability of conformance:

$$
\begin{aligned}
& \max p(x)=p(y \in A \mid x, \text { data }) \\
& \text { subjet } \quad \text { to }: x \in R,
\end{aligned}
$$

where $R$ is the region where the model is valid, which is usually taken as the experimental region defined by the design matrix $\boldsymbol{X}$.

According to [1] based on the typical multivariate regression model:

$$
Y=B x+e,
$$

where $\boldsymbol{B}$ is a $p x q$ matrix of regression coefficients and $\boldsymbol{x}$ is the qx1 vector of values $\boldsymbol{x}$ in which equation (4) is evaluated. In addition, the vector $\boldsymbol{Y}$ has a multivariate normal 
distribution with mean vector $\mathbf{0}$ and variance-covariance matrix $\Sigma$. To consider the uncertainty in model parameters $B$ y $\Sigma$ it can be used the posterior predictive density $f(y \mid x$, data). Using the classical noninformative prior joint distribution for $B$ y $\Sigma$ and the model of equation (3), the bayesian predictive density for $\boldsymbol{Y}$ can be obtained in closed form. The bayesian predictive density for vector values $\boldsymbol{x}$ where the function is evaluated, is given by a multivariate $t$ distribution with $\boldsymbol{V}$ degrees of freedom (df), as follows:

$$
f(y \mid x, \text { data })=\frac{\Gamma\left(\frac{v+p}{2}\right)}{(\pi v)^{p / 2} \Gamma\left(\frac{v}{2}\right)} \sqrt{|H|}\left\{1+\frac{1}{v}\left(y-\hat{B}^{\prime} x\right)^{\prime} H\left(y-\hat{B}^{\prime} x\right)\right\}^{-\frac{v+p}{2}},
$$

where

$$
\begin{gathered}
H=\left(\frac{v}{n-q}\right) \frac{\sum^{-1}}{1+x^{\prime}\left(X^{\prime} X\right)^{-1} x}, \\
p(x)=\frac{\text { Values in } A}{\text { Values generated }} .
\end{gathered}
$$

Here $p$ is the number of responses to optimize, $\boldsymbol{X}$ is the qxn matrix formed by the $\mathrm{n}\left(x_{i}\right)$ vectors of covariates, $\hat{B}$ is the least squares estimation of $\mathrm{B}, \Gamma($.$) refers to the$ gamma function, $\hat{\Sigma}$ is the usual estimator of $\Sigma$ calculated using the residuals of multivariate regression adjustment, $v=n-p-q+1$ and $n$ is the sample size.

According to [1] because equation 5, follows a multivariate t-distribution, is easy to simulate the $\boldsymbol{Y}$ values from this predictive density. [19] in his book Multivariate statistical simulation mentions that it is possible to simulate a random variable $\mathrm{t}$ - varied $\boldsymbol{Y}$, by simulating a multivariate normal random variable and an independent Chi-square random variable [1] If $\boldsymbol{W}$ is a normal random variable with mean vector equal to zero and variance-covariance matrix equal to $H^{-1}$ and additionally $\boldsymbol{U}$ is a Chi-square random variable which is independent of $\boldsymbol{W}$, then:

$$
Y_{j}=\left(\sqrt{v} W_{j} / \sqrt{U}\right)+\mu_{j} \quad \text { for } j=1, \ldots, p,
$$

where $\boldsymbol{Y}_{j}$ is the jth element of $\boldsymbol{Y}, \mathrm{W}_{\mathrm{j}}$ is the j-th element of $\boldsymbol{W}$ and $\hat{\mu}_{j}$ is the jth element from $\widehat{\mu}=\widehat{B} z(x)$. From the above mentioned by [1] it follows that $\boldsymbol{Y}$ follows a multivariate $\mathrm{t}$ distribution with $v$ degrees of freedom.

\section{Case Study}

To illustrate the operation of the methodology, the experimental data proposed by [20] are taken, which considers three design variables, $\mathrm{x}_{1}$ : reaction time, $\mathrm{x}_{2}$ : temperature and $\mathrm{x}_{3}$ : percentage of catalyst and two response variables, $\mathrm{y}_{1}$ : conversion rate and $\mathrm{y}_{2}$ : 
thermal activity using a central composite design with six central runs. In this case $\mathrm{x}_{1}$ was considered as noise variable $\left(\mathrm{x}_{\mathrm{n}}\right)$, so the optimization will be performed in relation to the control variables $\left(\mathrm{x}_{\mathrm{c}}\right) \mathrm{x}_{2}$ and $\mathrm{x}_{3}$. Data are shown in Table 1 .

Table 1. Experimental results.

\begin{tabular}{ccccc}
\hline & \multicolumn{2}{c}{ Control Variables } & \multicolumn{2}{c}{ Response Variables } \\
Reaction time & Temperature & \% Catalyst & Conversion rate & Thermal Activity \\
\hline$X_{n}$ & $X_{2}$ & $X_{3}$ & $Y_{1}$ & $Y_{2}$ \\
-1 & -1 & -1 & 74 & 53.2 \\
1 & -1 & -1 & 51 & 62.9 \\
-1 & 1 & -1 & 88 & 53.4 \\
1 & 1 & -1 & 70 & 62.6 \\
-1 & -1 & 1 & 71 & 57.3 \\
1 & -1 & 1 & 90 & 67.9 \\
-1 & 1 & 1 & 66 & 59.8 \\
1 & 1 & 1 & 97 & 67.8 \\
-1.682 & 0 & 0 & 76 & 59.1 \\
1.682 & 0 & 0 & 79 & 65.9 \\
0 & -1.682 & 0 & 85 & 60 \\
0 & 1.682 & 0 & 97 & 60.7 \\
0 & 0 & -1.682 & 55 & 57.4 \\
0 & 0 & 1.682 & 81 & 63.2 \\
0 & 0 & 0 & 81 & 59.2 \\
0 & 0 & 0 & 75 & 60.4 \\
0 & 0 & 0 & 76 & 59.1 \\
0 & 0 & 0 & 83 & 60.6 \\
0 & 0 & 0 & 80 & 60.8 \\
0 & 0 & 0 & 91 & 58.9 \\
\hline
\end{tabular}

Based on the information from the previous experiment the magnitude of subdivisions in the control factors to be used in the simulation to create a fine grid to generate response vectors using equation (7) is determined. These response vectors were used to calculate the corresponding desirabilities. In this case study we worked with a $11^{2}$ array, which means that 121 different combinations of control factors $x_{2}$ and $\mathrm{x}_{3}$ are used with the following levels for each variable:

$\{-1.682,-1.3456,-1.0092,-0.672,-0.3364,0,0.3364,0.6728,1.0092,1.3456,1.682\}$.

Once the number of combinations to be considered in the simulation is fixed, it is required to determine the number of runs to be simulated in each combination using equation (7) taking the adjusted regression models based on the original design. This time 10,000 vectors of two responses $\left(y_{1}, y_{2}\right)$ were generated in each one of the 121 combinations.

At this point it is also addressed the robustness of the process, considering that for each of the combinations of $x_{2}$ and $x_{3}$, while they remain fixed in the simulation of the 10,000 response vectors, the variable $\mathrm{x}_{1}$ takes a different value in each iteration, which is generated from a normal distribution $(0,0.1)$, whereby a vector of covariates $\mathrm{x}_{1}, \mathrm{x}_{2}$ 
and $\mathrm{x}_{3}$ is formed. With these 10,000 response vectors the desirabilities are calculated using equation 1 in each of the combinations of the control variables, comparing the values of the response variables against the corresponding specifications which are presented in Table 2.

Table 2. Specifications of the response variables.

\begin{tabular}{ccccc}
\hline Response & LSL & Target & USL & Kind of variable \\
\hline$y_{1}$ & 80 & 110 & - & The bigger the better \\
$y_{2}$ & 50 & 57.5 & 65 & The nominal is best \\
\hline
\end{tabular}

Once the individual desirabilities have been calculated the dimension of the problem is reduced by calculating $O D$ for each vector generated in each of the 121 combinations using Equation 2. In this case study four scenarios were proposed by changing the levels of importance of the response variables. In scenario $1\left(O D_{1}\right)$ the response variables are considered equally important, while in scenarios $2\left(O D_{2}\right), 3\left(O D_{3}\right)$ and $4\left(O D_{4}\right)$ is fictitiously considered that response variables have different importance, which is reflected in different weights in order to assess how the optimal point reacts to these weights. Table 3 shows the weights used for each response variable in each one of the proposed scenarios.

Using the $O D$ values, polynomials regression models were adjusted using Minitab 16 to represent the relationship between them and the control variables for the four scenarios under analysis. As an example, equation 8 shows the adjusted model for $\mathrm{OD}_{3}$.

The adjusted $\mathrm{R}^{2}$ for all four models were greater than $96 \%$ and for this reason the optimization (maximization) of these regression models was carried out. In this case, the optimization was realized with the help of Excel Solver using the Generalized Reduced Gradient method (GRG) to determine the best levels of the control variables with the maximum $O D$ for every one polynomial model. To initiate the search 15 values for control variables were used. The proposed optimal process operating condition and the $O D$ achieved in each model are shown in Table 4.

Table 3. Weights considered for the response variables in each scenario.

\begin{tabular}{lcc}
\hline & \multicolumn{2}{c}{ Weight } \\
\hline Scenario & $Y_{1}$ & $Y_{2}$ \\
\hline$O D_{1}$ & 0.5 & 0.5 \\
$O D_{2}$ & 0.6 & 0.4 \\
$O D_{3}$ & 0.8 & 0.2 \\
$O D_{4}$ & 0.2 & 0.8 \\
\hline
\end{tabular}

$$
\begin{aligned}
M O D_{3}= & 0.155+0.0708 x_{2}+0.0764 x_{3}-0.118 x_{2} x_{3}+0.320 x_{2}{ }^{2} \\
& -0.0971 x_{3}{ }^{2}+0.0144 x_{3}{ }^{3} x_{2}-0.0299 x_{2}{ }^{4}+0.0208 x_{3}{ }^{4} \\
& -0.00812 x_{3}{ }^{4} x_{2}{ }^{2} \\
R^{2}= & 96.9 \%
\end{aligned}
$$


Jorge Limon-Romero, Yolanda Baez-Lopez, Diego Tlapa, Mitzy Pory-Lugo, Ivan Rodriguez-Borbon

Table 4. Desirability Values in every optimal process operating conditions.

\begin{tabular}{lccccc}
\hline \multirow{2}{*}{ Scenario } & & Weights & \multicolumn{3}{c}{ Optimal values } \\
& $Y_{1}$ & $Y_{2}$ & $X_{2}$ & $X_{3}$ & \\
\hline$O D_{1}$ & 0.5 & 0.5 & 1.682 & -0.6055 & 0.8022 \\
$O D_{2}$ & 0.6 & 0.4 & 1.682 & -0.5710 & 0.8513 \\
$O D_{3}$ & 0.8 & 0.2 & 1.682 & -0.5208 & 0.9737 \\
$O D_{4}$ & 0.2 & 0.8 & 1.5073 & -0.7756 & 0.7140 \\
\hline
\end{tabular}

Finally, optimal proposed process operating conditions are tested using a matlab program very similar to that used in the initial simulation with a small change to allow that the control variables had fixed values, which corresponded to the optimum values proposed by the Solver; to do this 10,000 iterations were used. The simulation was repeated 30 times in order to know how the respective individual desirabilities for every response variable changed. With these values graphical comparisons of the two response variables for the four scenarios were made to see if there was a change on their desirabilities due to the different weights they had in each scenario. Figure 2 shows the behavior of the desirability associated to $Y_{l}\left(D_{l}\right)$ in the four scenarios analyzed. The graph shows that generally $D_{l}$ has the highest values in scenario $O D_{3}$, which is caused because this response variable had a weight of 0.8 , while in the other scenarios $Y_{l}$ had lower weights. The opposite occurs in $O D_{4}$ because is in this scenario where the lowest weight was given to this response variable, reflected in turn in the lowest values of $D_{l}$.

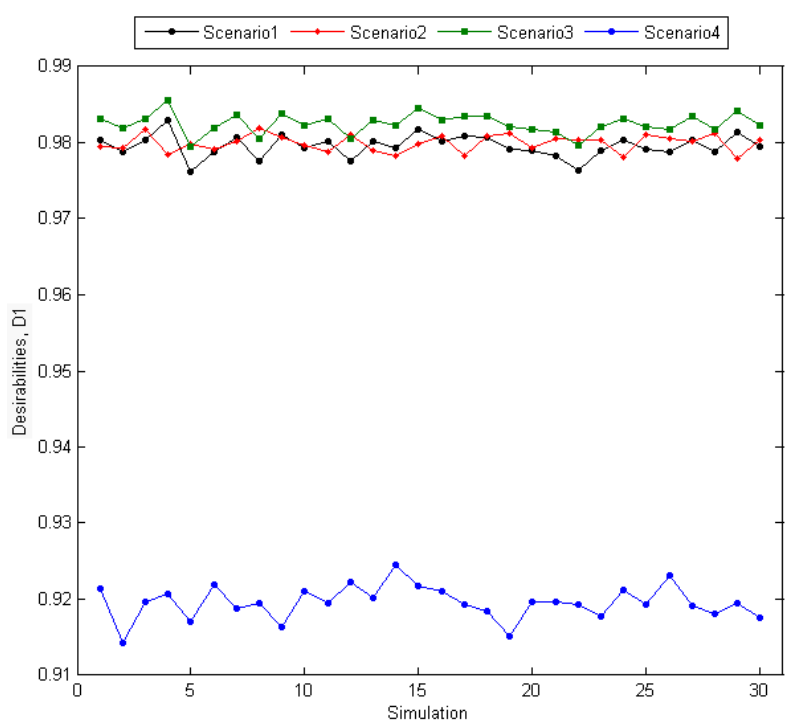

Fig. 2. Comparison of $D_{l}$ values in the four scenarios.

In the case of behavior of desirability associated to the variable $Y_{2}\left(D_{2}\right)$, the four scenarios can be seen in Figure 3. It is noted that in $D_{2}$ the highest desirabilities were in the scenario $\mathrm{OD}_{4}$ in the 30 repetitions of validation, this is because in this scenario 
the highest weight $(0.8)$ was given to this variable. In general, similar conclusions than those presented in figure 2 can be made with $D_{2}$ going up or down depending on the weights given to $Y_{2}$ in every scenario.

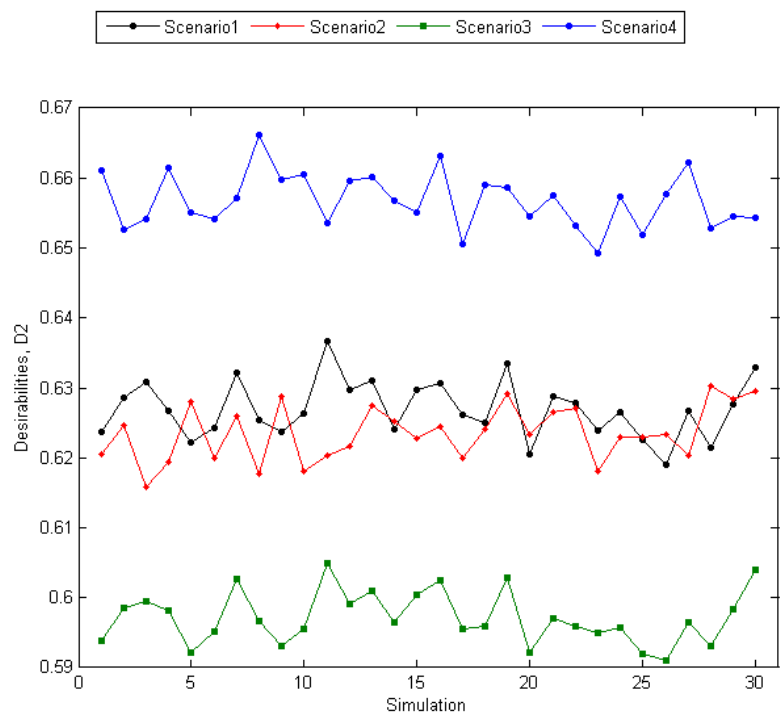

Fig. 3. Comparison of $\mathrm{D}_{2}$ values in the four scenarios.

With the previous figures the hypothesis that the best results achieved in the different proposed scenarios tend to favor the response variables with highest weights is confirmed graphically.

Table 5. Confidence intervals for the desirabilities in each response variable.

\begin{tabular}{|c|c|c|c|c|c|c|}
\hline \multirow{2}{*}{ Scenario } & \multicolumn{2}{|c|}{ Weigths } & \multicolumn{2}{|c|}{ Average } & \multicolumn{2}{|c|}{ Confidence interval (95\%) } \\
\hline & $Y_{1}$ & $Y_{2}$ & $D_{1}$ & $D_{2}$ & $D_{1}$ & $D_{2}$ \\
\hline$O D_{1}$ & $(0.5)$ & $(0.5)$ & 0.9795 & 0.6269 & $\begin{array}{c}(0.978972, \\
0.980101)\end{array}$ & $\begin{array}{c}(0.625400, \\
0.628480)\end{array}$ \\
\hline$O D_{2}$ & $(0.6)$ & $(0.4)$ & 0.9799 & 0.6236 & $\begin{array}{c}(0.979508, \\
0.980352)\end{array}$ & $\begin{array}{c}(0.622063, \\
0.625037)\end{array}$ \\
\hline$O D_{3}$ & $(0.8)$ & $(0.2)$ & 0.9825 & 0.5972 & $\begin{array}{c}(0.981972, \\
0.982994)\end{array}$ & $\begin{array}{c}(0.595745, \\
0.598568)\end{array}$ \\
\hline$O D_{4}$ & $(0.2)$ & $(0.8)$ & 0.9196 & 0.6568 & $\begin{array}{c}(0.918730, \\
0.920397)\end{array}$ & $\begin{array}{c}(0.655318, \\
0.658302)\end{array}$ \\
\hline
\end{tabular}

Table 5 shows the $95 \%$ confidence interval estimates of individual desirabilities, based on the 30 values obtained during validation. With these intervals it can be seen that there is a statistically significant difference in individual desirabilities of the response variables in the four scenarios presented. In general, the limits of the intervals are shifted up as the weights are increased and on the contrary, the limits decreased when the weights also decreased. Thus, according to these results the proposed 
methodology reacted according to the weights given to each response variable, searching the process operating conditions, where the variables with the highest weights behave better.

\section{Conclusions}

According to the results achieved in this and another case study not reported in this study it is possible to conclude that the methodology proposed by [1] and supplemented by [2] works well with the proposed modification to consider that the response variables have different weights, which in turn can modify the optimum results achieved, searching process configurations with better behavior in the variables with the highest weights. For this reason it makes possible to analyze scenarios where response variables are not equally important, so that the original methodology becomes more flexible and attractive in many practical cases, since in its original version is not designed to incorporate these considerations. Other advantage is that with the proposed modification to incorporate the desirability function, a problem can be solved using different combinations of weights on the response variables, which would provide to engineers and researchers different solutions with respect to the proposed optimal process operation condition, which can enrich decision making.

\section{References}

1. Peterson, J. J.: A Posterior Predictive Approach to Multiple Response Surface Optimization. Journal of Quality Technology, pp. 139-153 (2004)

2. Miro-Quesada, G., Del Castillo, E., Peterson, J. J.: A Bayesian Approach for Multiple Response Surface Optimization in the Presence of Noise Variables. Journal of Applied Statistics, pp. 251-270 (2004)

3. Ames, A. E., Matucci, N., Macdonald S., Szonyi, G., Hawkins, D. M.: Quality Loss Function for Optimization Across Multiple Response Surfaces. Journal of Quality Technology, pp. 339-346 (1997)

4. Ch'ng, C. K., Quah S. H., Low H. C.: Index $\mathrm{C}^{*} \mathrm{pm}$ in multiple response optimization. Quality Engineering, pp. 165-171 (2005)

5. Plante, R. D.: Process capability: a criterion for optimizing multiple response product and process design. IIE Transactions, pp. 497-509 (2001)

6. Derringer, G., Suich, R.: Simultaneous optimization of several response variables. Journal of Quality Technology, pp. 214-219 (1980)

7. Ortiz, F., Simpson, J. R., Pignatiello, J. J., Heredia-Lagner.: A Genetic Algorithm Approach to Multiple-Response Optimization. Journal of Quality Technology, pp. 432-450 (2004)

8. Lee, M. S., Kim, K. J.: Expected Desirability Function: Consideration of Both Location and Dispersion Effects in Desirability Function Approach. Quality Technology and Quantitative Management, pp. 365-377 (2007)

9. Chio, C.H., Hamada, M.: Analyzing Experiments with correlation multiple responses. Journal of Quality Technology, pp. 451-465 (2001) 
Optimization of Multiple Response Variables Using the Desirability Function and a Bayesian ...

10. Bahloul, B. Ali L., M., Sfar, S.: A novel Approach for the development and optimization of of self emulsifying drug delivery system using HLB and response surface methodology: Application to fenofibrate encapsulation. International Journal of Pharmaceutics, pp. 341-348 (2014)

11. De Figueiredo, A.K., Rodríguez, L.M., Riccobene, I.C., Nolasco, S.M.: Analysis of the Performance of a Dehulling System for Confectionary Sunflower Seeds. Food and Nutrition Sciencies, pp. 541-548 (2014)

12. Gutierrez, H., De la Vara, R.: Análisis y Diseño de Experimentos. México, Mc Graw Hill (2012)

13. Chiao, C. H., Hamada, M.: Analyzing Experiments With Correlated Multiple Responses. Journal of Quality Technology, pp. 451-465 (2001)

14. Vera, C. L., M. De Zan, M., Cámara, M. S., Goicoechea, H. C.: Experimental design and multiple response optimization. Using the desirability function in analytical methods development. Journal Talanta, pp. 123-138 (2014)

15. Ko, Y. H., Kim, K. J., Jun, C. H.: A new loss function-based method for multiresponse optimization. Journal of Quality Technology, pp.50-59 (2005)

16. Del Castillo, E., Montgomery, D.C., McCarville, D.R.: Of self emulsifying drug delivery system using HLB and response surface methodology: Application to fenofibrate encapsulation. International Journal of Pharmaceutics, pp. 341-348 (1996)

17. Simsek, B., Tansel, Y., Simsek, E. H.: A fullfactorial desing based desirability function approach for optimization of properties of C 40/50 concrete class. Journal of Mathematical and Computational Applications, pp. 330-339 (2013)

18. Zhang, X., Lu, X., Li, S., Zhong, M., Shi, X., Luo, G., Ding, L.: Investigation of 2,4-dichlorophenoxyacetic acid adsorption onto MIEX resin: Optimization using response surface methodology. Journal of the Taiwan Institute of Chemical Engineer, pp. 1835-1841 (2004)

19. Jhonson, M. E.: Multivariate Statistical Simulation: A Guide to Selecting and Generating Continuous Multivariate Distributions. USA: John Wiley \& Sons, Inc, (1987)

20. Myers, R. H., Montgomery, D. C.: Response Surface Methodology Process and Product Optimization Using Designed Experiments. New York: Wiley lnterscience (1995) 